Mots. Les langages du politique

\title{
La ligne bleue des Vosges
}

The blue line of the Vosges

La Linea azul de los Vosgos

\section{Aude Dontenwille-Gerbaud}

\section{OpenEdition}

\section{Journals}

Édition électronique

URL : https://journals.openedition.org/mots/21665

DOI : $10.4000 /$ mots. 21665

ISSN : 1960-6001

\section{Éditeur}

ENS Éditions

\section{Édition imprimée}

Date de publication : 15 septembre 2014

Pagination : 13-26

ISBN : 978-2-84788-543-9

ISSN : 0243-6450

\section{Référence électronique}

Aude Dontenwille-Gerbaud, «La ligne bleue des Vosges ». Mots. Les langages du politique [En ligne],

105 | 2014, mis en ligne le 15 septembre 2016, consulté le 23 avril 2022. URL : http://

journals.openedition.org/mots/21665; DOI : https://doi.org/10.4000/mots.21665 


\section{La ligne bleue des Vosges}

Selon les générations, l'expression peut rappeler quelque ancienne leçon d'histoire sur la Troisième République endeuillée par la perte de l'AlsaceMoselle en 1870, renvoyer à un combat politique contre une certaine idée de la France, ou plus récemment évoquer simplement un slogan publicitaire pour un doux voyage romantique. L'expression, devenue courante, mérite une étude approfondie pour en préciser la nature hautement problématique et politique. En quoi le bleu de la ligne des Vosges pose-t-il question?

Cette expression est forgée par Jules Ferry. Il est coutume de lire qu'elle apparaît dans son testament, rendu public après son décès le 17 mars 1893 : "Je désire reposer dans la même tombe que mon père et ma sœur, en face de cette ligne bleue des Vosges, d'où monte jusqu'à mon cœur fidèle la plainte touchante des vaincus» (Ferry, 1893, t. VII, p. 437). Elle est reprise instantanément par toute la presse. Qu'en est-il donc de cette évocation de la couleur, en quoi sa place dans un testament la rend-elle particulièrement remarquable, quel lien entre cette couleur et le pathos de la phrase, pourquoi un tel succès? Cette expression relève-t-elle de considérations de type «cognitivo-perceptuel » en cette fin de xıx siècle empreinte de romantisme? Comment se mêlentelles aux considérations socio-historiques qui ont tissé un lien étroit entre la République et la couleur bleue?

Cet article propose, dans une démarche d'analyse du discours par l'historien, d'effectuer une recherche sur le trajet de cette expression à partir d'un corpus daté de mars 1893 (la mort de Jules Ferry) à janvier 1920 (les problématiques politiques changent après le Traité de Versailles). Trois types de publication seront retenus. Nous avons effectué tout d'abord une revue de presse à propos de quelques événements emblématiques : les obsèques de Jules Ferry le 17 mars 1893, le $3^{\text {e Tour }}$ de France cycliste de juillet 1905 qui aborde les Vosges pour la première fois, l'assassinat de Jean Jaurès le 31 juillet 1914, la déclaration de guerre du 3 août 1914, l'armistice du 11 novembre 1918, le traité de Versailles du 28 juin 1919. Les quotidiens dépouillés s’inscrivent dans une large palette politique ${ }^{1}$. Nous avons

1. L'Estafette est le journal de Jules Ferry, La République française le journal «opportuniste » de Léon Gambetta, La Lanterne celui de Rochefort, opposant de longue date à Jules Ferry; Le Figaro 
recherché ensuite (grâce au site Gallica) un ensemble de récits militaires ou à vocation patriotique, datés de 1898 à 1920. Enfin, le dernier type de publication concerne une grande voix, celle de Maurice Barrès. Ce représentant du néo-nationalisme fait-il référence à cette «ligne bleue des Vosges»?

Nous avons fait l'hypothèse que ce corpus permettait d'interroger la place de la couleur bleue dans le trajet de l'expression «ligne bleue des Vosges» en tant qu'outil argumentatif du discours politique français des années 1890-1920 et qu'elle participait ainsi pleinement de l'événement discursif (Guilhaumou, 2006) fondateur de la Troisième République.

Nous analyserons la scène d'énonciation (Maingueneau, 1998) au sein de laquelle se déploie le testament de Jules Ferry et comment cette référence au bleu pose de nombreuses questions qui s'inscrivent dans l'histoire de la Troisième République et l'héritage révolutionnaire. Nous chercherons ensuite à analyser les reprises, les modifications de l'expression. Nous y retrouverons la tension analysée par les historiens de la période, entre patriotisme et devoir de mémoire d'une part, volonté de sauvegarder la paix, voire pacifisme, d'autre part. L'expression suit-elle alors un développement de type formulaire (KriegPlanque, 2009) au sein duquel la couleur bleue fonctionnerait comme un marquage fort? Nous verrons enfin comment l'acte de langage, entre bleu France et bleu horizon, révèle de véritables enjeux axiologiques.

\section{Le choix du bleu par Jules Ferry}

Jules Ferry n'a pas attendu son testament pour faire référence au bleu. Il emploie déjà une expression presque similaire lors d'un discours prononcé le 30 juillet 1889 pour la remise des prix au collège de Saint-Dié. Il y évoque la nouvelle réforme militaire et les obligations de la conscription :

Dans une race militaire et sur cette frontière, cette obligation nouvelle est accueillie sans murmures. On peut en parler devant des mères lorraines. Oui, Mesdames, si la charge vous paraît lourde, si votre tendresse maternelle en tressaille et s'en inquiète, levez les yeux vers le ciel, du côté de l’Est, regardez la ligne bleue des Hautes Vosges... (Ferry, 1893, t. VII, p. 366)

En 1889, la géographie se veut plus précise que celle du testament : seules les Hautes Vosges forment une ligne bleue. La question de la frontière y est explicite. Ce n'est plus le cas dans le testament : «Je désire reposer dans la

représente la bourgeoisie républicaine conservatrice, La Croix - à l'époque - un lectorat antirépublicain, anti-dreyfusard, anti-vatican; Le Petit Journal s'adresse à un lectorat populaire qui s'en détache vers la fin du siècle quand il prend des positions antidreyfusardes puis nationalistes. Nous y avons ajouté L'Aurore, fondée par Clemenceau en 1897, L'Humanité, fondée par Jean Jaurès en 1904 et L'Action française, qui date de 1908. Soit 140 numéros de la presse nationale. 
même tombe que mon père et ma sœur, en face de cette ligne bleue des Vosges, d'où monte jusqu'à mon cœur fidèle la plainte touchante des vaincus » : l'expression semble à ce point essentielle à Jules Ferry qu'elle figure à la fin d'une dizaine de lignes, après les legs qu'il souhaite faire à sa famille. En reprenant les catégories élaborées par Dominique Maingueneau, remarquons que si la scène générique du discours est celle du testament, la scénographie qui s'impose d'entrée de jeu, par son appel aux sensations (le bleu) et à l'émotion, se veut poétique, tout en ayant comme visée une scène englobante politique. L'identité des partenaires de l'énonciation (la famille de Jules Ferry d'abord, mais également la grande famille qu'est la France vaincue) va de pair avec un lieu de l'énonciation (la ligne bleue des Vosges) et un moment d'énonciation (la mort) dans une perspective d'action sur autrui (le choix de la sépulture qui invite au recueillement national, voire au pèlerinage). Mais pourquoi le bleu, plutôt que le noir ou le vert? Des goûts et des couleurs, on ne peut disputer... Comme l'analyse Vincent Moriniaux (Moriniaux, 2001, p. 371), toutes les montagnes, dans le lointain, apparaissent plus ou moins bleutées. Ce phénomène physique n'est pas propre aux Vosges, ni même propre à la sapinière. II reste d'ailleurs fort difficile à photographier, ce qui explique l'absence de cartes postales autres qu'humoristiques de cette fameuse « ligne bleue des Vosges». Tout le monde en parle mais personne ne l'a jamais vue. Jules Ferry voit donc cet horizon «bleu » et l'expression se veut d'abord celle d'un individu et de son expérience visuelle. Sur une toile de la fin du XIxe siècle du peintre Henri Rovel, la vue du chalet acheté à Foucharupt par Jules Ferry laisse apercevoir une ligne bleue qui tourne facilement au violet...

Pour autant, cette métaphore fonctionne-t-elle comme un euphémisme (Jamet, Jobert, 2010), une façon de dire sans dire la défaite de 1870, la perte de l'Alsace-Moselle dont Gambetta proposait «d'y penser toujours, n'en parler jamais...»?

Le 22 mars 1893, L'Estafette, le journal de Jules Ferry, commente l'expression devenue célèbre instantanément :

Comme une violette cueillie aux fleurs d'un bouquet, cette phrase du testament reproduite par la presse entière n'associe-t-elle pas, plus que les discours, Jules Ferry à sa patrie, ne le venge-t-elle pas des reproches iniques qui furent adressés à son loyalisme? Je désire reposer dans la même tombe que mon père et ma sœur, en face de cette ligne bleue des Vosges, d'où monte jusqu'à mon cœur fidèle la plainte touchante des vaincus. Tous ceux dont l'âme est douce, pétrie d'un peu d'espérance et de fierté, voilée d'un peu de poésie, - c'est-à-dire en ce pays qui demeure rêveur et chevaleresque, la très grande majorité -, tous comprendront la mélancolie hautaine de l'homme qui, ignorant le sort futur de la terre lorraine, n'a point voulu refuser de prendre sa part de deuils, peut-être éternels ?²

2. L'Estafette, 22 mars 1893, p. 2. 
La ligne bleue des Vosges s'associe à la violette... Dans un cas, un adjectif, dans l'autre un nom. Dans la mesure où le bleu semble avoir ici une fonction de catégorisation, son statut nettement épithétique ne peut admettre de modification de degré (Molinier, 2006). Le bleu catégorise en elle-même la «ligne des Vosges », elle est sa propriété inhérente (Dubois, 2009). Mais dans un élan métadiscursif, le journaliste de L'Estafette l'associe à une autre couleur, cette fois nominalisée dans la fleur violette, symbole de délicatesse et de timidité. Le commentateur invite donc à une lecture qui s'oppose à toute une tradition du bleu national, attribué aux événements guerriers. Le violet, c'est aussi celui des deux provinces perdues sur les cartes de France des écoles primaires. L'Estafette associe le choix poétique du bleu de Jules Ferry à un tempérament rêveur, chevaleresque, mélancolique. Cependant, l'ethos discursif présenté ici semble à première vue paradoxal pour un homme de gouvernement. L'évocation des couleurs amène le lecteur dans un registre sensible, souligné par le commentateur comme une forme d'expérience poétique «audelà du discours », qui rappelle quelque peu le romantisme allemand particulièrement sensible au bleu (Pastoureau, 2002 : 121). Peut-on dès lors évoquer une euphémisation, principe discursif qui refuse les termes dysphoriques et le registre sensible (Bonhomme, 2005) ? L'emploi de l'euphémisme construit une posture particulière du locuteur. Caractéristique de la prudence (au sens de la phronesis d'Aristote), l'euphémisation renvoie une image consensuelle. L'ethos discursif porté par la « ligne bleue des Vosges» rejoint ici l'ethos institutionnel d'un homme d'État qui n'a jamais revendiqué de politique agressive à l'égard de l'Allemagne, et jamais évoqué le moindre désir de «revanche». L'ethos discursif souligné par L'Estafette trouve alors tout son sens.

Une revue de presse, au moment des obsèques de Jules Ferry, permet de mettre en évidence l'importance que revêt aussitôt cette expression. Les divers journaux rendent compte (en faisant souvent erreur) de sa place au sein du testament. Nous avons là une réécriture journalistique révélatrice. La seconde moitié du XIX'e siècle marque l'entrée dans l'ère moderne des médias, au sens où peuvent l'analyser Gilles Feyel (2007) ou encore Dominique Kalifa (2001). Au travers d'un flot croissant de journaux, de revues, de magazines, et des informations qu'il charrie, l’individu moderne est appelé à se définir. Dominique Kalifa parle d'une fonction d'acculturation. La référence au bleu y participe donc pleinement. La République française, par les voix de Joseph Reinach et Eugène Spuller, fait part de sa grande tristesse. Le dimanche 19 mars, le testament est cité, sans commentaire, à la rubrique «Les Obsèques». Elle est conjointe à une rubrique signée d’Eugène Spuller qui présente Jules Ferry comme un « révolutionnaire, de la bonne façon et dans la meilleure acception du mot ». La ligne bleue des Vosges du testament se retrouve par là même associée au bleu déclaré «national » en $1790 . .$. Non pas un bleu sombre, tournant au violet, mais le «bleu France» de la Garde nationale au service des idées révolutionnaires. Le PetitJournal insiste, lui, 
sur le calme du défunt et l'admirable courage de sa femme, pour conclure par la citation, là encore sans commentaire mais qu'il considère comme la première du testament. Le Figaro, quant à lui, cite le testament pour expliquer que Jules Ferry sera enterré à Saint-Dié et non à Paris. La phrase est considérée comme la dernière du testament. Les deux journaux se trompent mais l'insistance sur l'expression révèle l'importance de ce bleu dans la construction du «roman national». «La ligne bleue des Vosges» ne peut s'analyser sans référence à l'interdiscours que forment les romans nationaux d’Erckmann-Chatrian par exemple, «La dernière classe, récit d'un petit Alsacien », extraits des Contes du lundi d'Alphonse Daudet, ou encore le célèbre Tour de la France par deux enfants de G. Bruno. Pour un lecteur de l'époque, le testament de Jules Ferry fait écho aux dernières paroles du père mourant des deux petits alsaciens du célèbre livre de lecture courante. Le bleu de la ligne des Vosges se mêle alors au bleu du ciel.

La main froide de l'agonisant serra d'une faible étreinte les mains des deux enfants réunies dans la sienne, puis ses yeux se tournèrent vers la fenêtre ouverte par où se montrait un coin du grand ciel bleu : il semblait chercher par-delà l'horizon cette frontière reculée de la chère patrie où il n'irait pas; mais où ses deux fils, sans appui désormais, lui promettaient de se rendre. (Bruno, 1877, p. 10)

Toutefois, une partie de la presse dénie à Jules Ferry le droit à cette expression, le bleu ne renvoyant alors pas aux mêmes valeurs nationales. Dans le journal La Croix, par exemple, «la ligne bleue » figure en gros caractères et en majuscule, et forme le titre de l'article en première page. Le terme «Vosges» a disparu. Le bleu incarne donc ici à lui seul la défaite, la question de l’AlsaceMoselle, la frontière.

Et je le laisserais dans la mort, régler seul son compte avec Dieu, si, par son testament public, il n'engageait l'avenir en écrivant, “pour la postérité”, une phrase que je ne comprends pas : “Je veux, dit-il reposer à Saint-Dié, en face de la ligne bleue des Vosges et m'associer toujours à la plainte mélancolique des vaincus!” Encore une fois, cette phrase, nous ne la comprenons pas. 3

Suit une longue diatribe contre Jules Ferry, celui qui a cherché l'alliance avec Bismarck, celui qui a attisé les haines religieuses, et qui se termine par :

Non! Ce n'est pas à Saint-Dié qu'il doit reposer! La ligne bleue des Vosges est un cadre trop gracieux, trop religieux pour sa haineuse figure. Sainte Odile est trop près; trop de prêtres y passent, trop de cloches y sonnent, trop d'enfants y prient.

Le bleu n'est plus ici ni romantique, ni révolutionnaire, mais associé à la tradition chrétienne, il rappelle le célèbre «bleu de Saint-Denis» mis au point par les maîtres verriers vers 1140 et qui servira ensuite de référence à tous les bâtisseurs de cathédrales (Pastoureau, 2002).

3. La Croix, 25 mars 1893, p. 1. 
Toute la presse s'empare donc de l'expression de Jules Ferry, qui devient célèbre en elle-même, parfois pour des raisons diamétralement opposées. Les considérations cognitivo-perceptuelles rejoignent d'évidence les considérations socio-historiques. Ainsi que l'analysent Paul Bacot et Sylvianne RémiGiraud, l'espace de référence, source des transferts métaphoriques, fonctionne comme un réservoir «de traits et de propriétés dont la capacité expressive de politisation des conflits est en attente d'exploitation » (Bacot, Rémi-Giraud, 2002). En cette année 1893, la référence au bleu oppose encore héritiers d'une histoire de France chrétienne et héritiers de la Grande Révolution. Elle participe donc de cet événement discursif fondateur de la Troisième République. Quelques années plus tard, au tournant du siècle, puis lors de la Première Guerre mondiale, la référence au «bleu » met en scène une autre forme de conflit.

\section{Le bleu, enjeu d'une formule entre 1893 et 1920}

Une fois Jules Ferry inhumé, notons tout d'abord l'absence de cette expression dans la presse, que ce soit en 1905, en 1914, en 1918-1919. Lors de la troisième édition du Tour de France, en juillet 1905, la course s'attaque pour la première fois à l'ascension d'un massif, en l'occurrence le col du Ballon d'Alsace. Gilles Fumey analyse le «Tour de France comme l'un des plus prestigieux et des plus géographiques monuments de l'identité française» (Fumey, 2006, p. 388) montrant comment son tracé dessine une appropriation des frontières profondément ancrée dans la mémoire nationale. Curieusement, les quotidiens qui mentionnent le Tour de France 1905 n'évoquent absolument pas la question de l'Alsace-Moselle. Nulle «ligne bleue des Vosges» dans leurs commentaires. On ne retrouve pas non plus l'expression dans la presse après l'assassinat de Jean Jaurès le 31 juillet 1914, au moment de la déclaration de guerre du 3 août 1914, ni même lors de l'armistice du 11 novembre 1918 ou encore du traité de Versailles du 28 juin 1919. Dans le journal La Croix du 18 novembre 1914, Pierre L'Ermitte (l'éditorialiste qui refusait à Jules Ferry son testament en 1893), ne voit plus que simple sapinière : il décrit «le Mont Saint-Odile d'où l'on voit plus de 400 villages posés comme d'adorables joujoux sur l'immense tapis des prairies à l'ombre des forêts de sapins »4, comme si Metz, Strasbourg, l'Alsace tout entière étaient redevenues françaises en ces tout premiers mois de guerre. "La ligne bleue des Vosges» a en quelque sorte disparu. Ne peuton évoquer une forme d'autocensure qui fonctionnerait en discours comme un tabou dont il convient d'interroger la dimension axiologique? Derrière l'acte de langage apparent de cette presse, s'exprime sans doute un acte de requête : celui d'une forme d'union sacrée à propos du souvenir des provinces perdues.

4. La Croix, 18 novembre 1914, p. 1 
Il n'en est pas de même des récits militaires ou patriotiques. Certains reprennent et détournent l'expression de Jules Ferry.

Et quand il a plu fort en ces jours de naissant automne, quand les fonds d'horizon sont bien lavés, le curé d’Essey peut voir avec sa longue-vue toujours braquée sur les choses et sur les gens, le pays lorrain à des lieues et des lieues, jusqu'à ces monts vallonnés, aux vastes chaumes pelées qui sont nos Vosges, l'éternelle ligne bleue entre Moselle et Rhin. (Badel, 1916)

La ligne bleue est ici qualifiée d'«éternelle». Émile Badel écrit en octobre 1915 pour défendre les Monts sacrés de la Lorraine, mêlant patriotisme et foi catholique. La longue-vue, métaphore de l'attention portée à la question «Alsace-Lorraine», semble une critique en creux de ceux qui oublient les «choses et les gens», en d'autres termes, une politique républicaine pas assez clairement revancharde. D'autres n'ont même plus besoin de citer les Vosges. Ainsi de cet hommage aux chasseurs alpins :

Aussi, rompus à la fatigue, habitués comme leurs camarades de l'Est à la rudesse de la vie militaire, les diables bleus aux jarrets de fer ne furent pas au-dessous de leur renommée. Sortis des plaines de l'Alsace où ils avaient vaincu, ils s'abattirent sur les Vosges un beau matin de 1914, se retrouvant là dans leur élément. Ce qu’il en coûta à l'Allemand de se mesurer avec eux, Dieu seul peut le dire; mais nous savons ce que fut la défense de la ligne bleue sacrée par leur bataillon héroïque... (Vernines, 1919, p. 26)

La ligne bleue est devenue évidence, un implicite qui échappe à la contestation, tant le bleu fait partie d'un savoir partagé (Amossy, 2000), celui de la mémoire des provinces perdues, ligne bleue ici qualifiée de sacrée, sans doute en référence à «l'amour sacré de la patrie » de l'hymne national.

Mais dans ce récit de quatre pages sur la mobilisation, la ligne bleue n'est plus simplement «horizon»:

La ligne bleue...

4 août 1914

Il y a dans tous ces détails un sublime simple, fort et contagieux. Une force énorme qui s'assemble sans un bruit, un péril qui se dresse sans une crainte, voilà ce qui est la France d'aujourd'hui. Ni forfanterie, ni agression : cette fois c'est là le lot des autres. Et c'est ainsi qu'on mérite de pouvoir espérer, et c'est ainsi qu'un immortel «quand même», plus éloquent que nul autre, semble venir à nous de la ligne bleue des Vosges... (Hepp, 1916, p. 3)

Le mouvement s'inverse, non plus celui du regard qui confère le bleu à la ligne d'horizon, mais le lieu d'où part l'espoir, le bleu comme source de force. Dans notre corpus, nous trouvons donc «ligne bleue des Vosges », "ligne bleue des Hautes Vosges», «ligne bleue», «ligne des Vosges», «ligne bleue sacrée», "éternelle ligne bleue», «face à la ligne bleue des Vosges», "audelà de la ligne bleue»... Cette diversité des variantes est-elle le témoignage 
d'une activité de fonctionnement formulaire dans une véritable dynamique de débat? (Krieg-Planque, 2009). La «formule» cristallise des enjeux politiques et sociaux qu'elle contribue dans le même temps à construire, un événement de discours. Cela suppose une stabilité du signifiant, condition matérielle de reprise et de circulation de la formule. Quelles que soient les variantes et les connotations historiques auxquelles renvoie l'évocation du bleu, le signifiant reste bien la frontière entre la France et l'Allemagne, symbole de la défaite de 1870 et des provinces perdues. Il y a bien construction d'un événement de discours dans la mesure où la politique internationale et les stratégies militaires ne font pas consensus. Jules Ferry lui-même, dans un mouvement quelque peu paradoxal, évoquait cette tension : «Au nom d'un chauvinisme exalté mais à courtes vues, devions-nous acculer la politique française dans une impasse, et, les yeux fixés sur la ligne bleue des Vosges, laisser tout se faire, tout s'engager, tout se résoudre, sans nous, autour de nous? (Ferry, 1890, p. 51) C'est vraisemblablement la raison du choix éditorial d'un journal comme Le Figaro, le 18 juin 1919. À propos de la signature du traité de Versailles, le quotidien mentionne la «ligne des Vosges», titre de la première page. En un tel jour, l'absence de la couleur bleue s'apparente à un véritable choix politique du Figaro qui refuse ici la mémoire de Jules Ferry, «Ferry Tonkin », celui qui aurait abandonné l'esprit de la revanche pour le rêve colonial.

\section{Bleu horizon contre Bleu France?}

Nous retrouvons trace de cet événement de discours dans l'œuvre de Maurice Barrès. Il est coutume d'associer la grande voix du nationalisme français à la politique de la revanche. Pour autant, l'auteurn'a jamais employé l'expression de Jules Ferry. L'Appel au Soldat (1900), dans un grand élan poétique sur les Vosges, décrit le coucher de soleil sur les contreforts; Les Déracinés : le roman de l'énergie nationale (1911) se contente d'évoquer les Vosges; L'âme française et la guerre (1915-1920) se réfère à la crête et la trouée des Vosges ; Le génie du Rhin (1921) ne parle que des bastions de l'Est. En revanche, Scènes et doctrines du nationalisme (1902) présente la «ligne des Vosges» et non pas la trouée, la crête ou les sommets. Pas de bleu pour autant : citer Jules Ferry n'est pas possible pour le représentant d'un néo-nationalisme toujours virulent contre l'Allemagne, toujours porteur de l'esprit de revanche. Les amitiés françaises (1903) évoquent toutefois le bleu des Vosges, mais dans une formulation autre :

Il était tellement connu et admiré qu'un jour on le fit duc de Lorraine. Il devint ainsi le chef, non plus seulement du comté de Vaudémont, mais de tous les pays que tu peux voir, à droite, à gauche, aussi loin que ton regard s'étende [...] un autre jour, comme des bandes voulaient passer ces montagnes bleues, les Vosges, que tu vois là-bas, pour se jeter sur la France, il se mit en travers. (Barrès, 1903, p. 125) 
Nous sommes là dix ans après le décès de Jules Ferry et la publicité autour de son testament. "Ces montagnes bleues, les Vosges» ne peut être qu'un choix implicite que chaque lecteur comprendra. Si ce n'est que le duc de Lorraine est considéré comme un sauveur de ces montagnes bleues. Jean-Jacques Becker montre comment, pour le néo-nationalisme de la Belle-Époque, le vrai souci n'est pas seulement la question de l'Alsace-Moselle mais aussi celle d'une décadence intérieure de la "gueuse» (Becker, 2001). En ce sens, adhérer au bleu de Jules Ferry, au bleu de l'histoire révolutionnaire est proprement impossible. La Colline inspirée (1913) évoque le bleu : "On songe à ce lac bleu des Vosges dont les eaux glacées avaient infatué Charlemagne. Le vieil empereur ne pouvait plus en détacher son esprit, son regard. » (Barrès, 1913, p. 301) Ce n'est pas une «ligne» mais un lac qui captive un empereur. Le vieil homme semble y perdre la raison. Comme l'analyse Zeev Sternhell, la droite révolutionnaire voue un culte à l'affectivité, à l'instinct contre la rationalité, à la force physique contre l'intellectualisme. La déroute de 1870 est d'abord celle d'une société issue des Lumières, de la Révolution française et des vieilles valeurs républicaines (Sternhell, 1978, p. 86). Implicitement, le bleu du lac renvoie à ces origines mystérieuses et mythiques des Vosges. Dans un opuscule daté de 1905 de Paul Flamant, préfacé par Maurice Barrès, le bleu s'étend à toute la France : «À l'est, au fond de la plaine de Kehl, la Forêt-Noire ondule sur le ciel lourd de la Souabe; à l'ouest, le ruban déchiré des Vosges laisse apercevoir une France toute bleue, sous un ciel plus léger.» (Flamant, 1905, p. 14) Le «ciel lourd de la Souabe» et la Forêt-Noire renouent avec la vision mythique d'une Allemagne ennemie, celle qui remonte à l'antiquité de Tacite où la forêt de Germania ne pouvait engendrer que barbarie. Ce n'est plus la ligne des Vosges qui est bleue, parce que le ruban est déchiré : la France est implicitement bleue, couleur du ciel et non de la sapinière.

C'est pourtant ce bleu du ciel, qui sera dénommé, dix ans plus tard en 1915 «bleu horizon », celui destiné à rendre l'uniforme «bleu national» de 1914 plus discret. Même si le peintre lorrain Louis Guingot invente la première veste de camouflage dite «léopard» en septembre 1914, le bleu résiste. Selon Frédéric Thiery, au-delà des questions matérielles et économiques, l'idée même de camouflage était incompatible avec la pensée française de la guerre et le code d'honneur de la société militaire de l'époque : force, bravoure, « voir et se faire voir» (Thierry, 2007). Dans le syntagme bleu horizon se joue la tension énonciative entre une volonté pragmatique de résistance à l'Allemagne (un uniforme peu voyant à l'horizon) et l'affirmation d'une revanche sur la défaite de 1870 (l'horizon de la ligne bleue des Vosges). Maurice Barrès se retrouve luimême face à une jeunesse avide d'action, avide de «voir et se faire voir». Les jeunes gens d'aujourd'hui (1913), signé Agathon, font remonter la question nationale : «Que la mémoire fut demeurée fidèle chez les jeunes gens de l'Est, que parmi eux l'armée recrutait de nombreux officiers, il n'était à cela rien de 
surprenant : de Sedan comme de la ligne bleue des Vosges, leur cœur "entend toujours la plainte douloureuse des vaincus". » (Agathon, 1913, p.175) C'est la plainte des vaincus qui est soulignée ici dans la citation de Jules Ferry. La ligne bleue des Vosges n'est qu'un lieu au même titre qu'un autre, Sedan. Si ce n'est que Sedan est une défaite militaire de l'Empereur Napoléon III. La « ligne bleue des Vosges » en devient donc une défaite implicitement attribuée à la Troisième République et à l'un de ses fondateurs, Jules Ferry.

Au sortir de la guerre, le bleu horizon l'a en quelque sorte emporté sur le bleu national. Dans l'acte de langage qui consiste à nommer «Chambre bleu horizon » la nouvelle Chambre des députés du 16 novembre 1919, se joue une vision de la République. Ce n'est pas seulement la présence, parmi les députés, de nombreux anciens combattants qui lui confère ce surnom. De fait, cette nouvelle Chambre est la plus marquée à droite de toutes celles que la République a connues depuis 1875. Le bleu national issu de l'héritage révolutionnaire ne peut plus se dire. La «ligne bleue des Vosges » fonctionne donc bien comme une «formule», référent social indiscutable de toute la Belle Époque, que ce soit pour la citer, s'en saisir et la modifier ou même l'occulter. À un moment donné, dans un espace socio-politique donné, la formule se caractérise par son aspect dominant (Krieg-Planque, 2009, p. 93).

Pour conclure, essayons, par un rapide survol contemporain, de rechercher ce qu'il en est de cette expression dans un tout autre espace socio-politique. II semblerait que l'expression reste communément attachée à une vision fausse de la Troisième République, celle d'une époque revancharde, orchestrée par l'École laïque, gratuite et obligatoire. Les historiens ont montré que la question était infiniment plus complexe. L'analyse de discours de la formule inventée par Jules Ferry va dans ce sens. C'est sans doute ce qui fait hésiter le catalogue publié par le musée Pierre-Noël de Saint-Dié-des-Vosges dont le titre s'intitule Jules Ferry, de l'instruction publique à la «ligne bleue» des Vosges, mais qui évoque plus loin (p.52) «la ligne bleue des Vosges». La place des guillemets montre une difficulté à citer ce qui a fait mémoire : est-ce le bleu, est-ce les Vosges? «La plainte des vaincus », en tout état de cause, n'est pas retenue.

Aujourd'hui, cette «ligne bleue des Vosges », au-delà du mythe et des poncifs, est devenue argument de vente. Nous la retrouvons par exemple sur le site de l'Office du tourisme des Vosges sous le titre «La ligne bleue ». Pour autant, il n'est pas possible d'évoquer un topos. L'article décrit le massif des Vosges comme un ensemble de crêtes et de vallées où la nature s’impose. Rien dans ce cadre «naturel » ne renvoie à une quelconque frontière politique et historiques. Il faut aller à la rubrique «Histoire » pour que cette ligne bleue prenne sens par

5. http://www.vosges.fr/La-vie-en-Vosges/Tourisme-Vosges/Ligne-bleue.htm. Site visité le 12 juin 2013 
la citation du testament de Jules Ferry, à propos duquel il n'est toutefois fait aucun commentaire. En revanche, le site touristique conclut : "Une ligne bleue qui offre un magnifique paysage "horizontalisé" par l'alignement des sommets des montagnes arrondies ». Le néologisme «horizontalisé» se veut ambivalent entre un «horizon» (qui ne se dit plus) et un «horizontal» (pour souligner la spécificité du massif ancien arrondi, image de tous les manuels scolaires de géographie française). Le paragraphe est lui-même intitulé : "Jules Ferry écrit la Ligne Bleue de l'Histoire »... Sur la scène d'énonciation qui est celle d'un site touristique, ce titre et ce paragraphe peuvent être tout aussi bien compris comme une référence identitaire du département que comme un message ironique à propos d'une expression désormais désuète. Il serait surtout intéressant de pouvoir interroger la réception de cette expression par les internautes qui visitent ce site électronique. Un autre site touristique évoque lui aussi cette évidence, mêlant registre de la perception et ancrage historique :

Territoire en vert et bleu, la ligne bleue des Vosges s'impose à l'œil comme ligne d'horizon. [...] ces ondulations douces, ces formes arrondies, chatoiements de verts profonds, çà et là ourlé d'un voile léger, nimbé d’une lumière tamisée qui confère au massif cette coloration bleutée. Chère à Jules Ferry devant laquelle il voulut être enterré, louée par les écrivains romantiques, la ligne bleue des Vosges sur laquelle court la route des crêtes, constitue un véritable balcon panoramique. Elle demeure la citation identitaire la plus forte du massif des Vosges. ${ }^{6}$

L'évocation de la ligne bleue associe ici une réalité visuelle essentialisée que chacun se doit d'observer, et une «citation» dont on ne connaît d'ailleurs pas l'auteur. Si l'on va au bout du raisonnement, celui dont l'œil ne sera pas en mesure de percevoir la ligne bleue ne pourra rien comprendre de l'identité du lieu. C'est tout le sens de la question patrimoniale qui est posé dans ces quelques lignes. Mais la ligne bleue des Vosges, c'est aussi, et plus légèrement, un club de golf de Colmar, qui organise des «week-ends raquettes à neige» sur les crêtes des Vosges. On peut supposer qu'il est difficile alors de «voir» la « ligne bleue» sous le manteau blanc...

En dehors de cette forme de récupération touristique, l'expression garde parfois, encore aujourd'hui, sa dimension politique. Le 25 juillet 2008, par exemple, Le Figaro pouvait titrer «La fin de la ligne bleue des Vosges». L'éditorial d'Étienne de Montety commençait ainsi :

Depuis cent cinquante ans, une géographie militaire s'était imprimée dans la tête des Français : elle formait la ligne bleue des Vosges. Notre mythologie intime ne quittait pas le Rhin des yeux. Sans remonter jusqu'aux Huns, battus aux champs Catalauniques, nos aïeux savaient que la menace venait de l'Est : Sambre et Meuse, La Rothière, Verdun, les vents mauvais arrivaient toujours des plaintes de l'Orient.

6. http://www.deodatie.com/theme.html?id=126. Site visité le 12 juin 2013. 
L'éditorialiste y décrit l'importance des garnisons installées sur cette ligne, y compris durant la guerre froide. Ce temps a vécu et l'éditorialiste s'alarme, pour les collectivités territoriales, mais aussi pour l'armée?. Le Figaro semble renouer avec sa ligne éditoriale des années 1900-1914, celle d'une critique de la politique de défense de la France. Un journal satirique et parodique semble lui répondre. Brave Patrie, "Le vrai journal des vraies valeurs de la France vraie » 8 , publié uniquement sur le Net, décide en 2009 de déposer le titre :

«Devant le peu d'empressement des entreprises françaises à défendre les valeurs nationales [...], Bravepatrie.com a décidé de reprendre le flambeau du patriotisme économique. Nous avons déposé au Bureau des Brevets Improbables l'un de nos symboles les plus forts, celui pour lequel le plus grand nombre de patriotes petits et grands ont versé leur sang : la Ligne Bleue des Vosges. »

Brave Patrie.com propose une lecture courante, mais caricaturale. Au-delà des clivages politiques, l'utilisation de cette expression révèle la permanence du débat sur l'identité du territoire national. Le terrain du politique constitue un poste d'observation privilégié pour analyser en discours la valeur d'un terme de couleur, le bleu, qui n'a certainement pas terminé de susciter le débat en France.

\section{Corpus}

\section{Jules Ferry}

1890, Le Tonkin et la Mère-Patrie. Témoignages et documents, Paris, Victor-Havard. 1893, Discours et opinions de Jules Ferry, Paris, Armand Colin, (7 volumes). 1914, Lettres de Jules Ferry, Paris, Calmann-Lévy.

\section{Presse}

(Mars 1893, juillet 1905, août 1914, novembre 1918, janvier et juin 1919)

L'Action française

L'Aurore

LaCroix

L'Estafette

Le Figaro

L'Humanité

La Lanterne

7. Le Figaro, 25 juillet 2008.

8. http://www.bravepatrie.com. Site visité le 12 juin 2013. 
Le PetitJournal

La République française

\section{Publications diverses}

Agathon, 1913, Les jeunes gens d'aujourd'hui, Paris, Plon.

BADEL Émile, 1916, Les monts sacrés de la Lorraine. Excursions et souvenirs, Nancy, Imprimerie Lorraine.

BARRÈs Maurice, L'appel au soldat, Scènes et doctrines du nationalisme, Les amitiés françaises : notes sur l'acquisition par un petit Lorrain des sentiments qui donnent un prix à la vie, Les Déracinés : le roman de l'énergie nationale, La colline inspirée, L'âme française et la guerre. L'Union Sacrée, Le génie du Rhin : les bastions de l'Est, ouvrages consultables sur le site Gallica de la BNF.

BRU LAT Paul, 1907, Histoire populaire de Jules Ferry, Paris, La Librairie mondiale.

BRUno G., 1877, Le Tour de la France par deux enfants, Paris, Belin.

Flamand Paul, 1905, Au poteau frontière, Paris, Sansot et Cie.

HePp Alexandre, 1916, Les cœurs embellis, 1914-1915, Paris, Charpentier.

VERNINES Pierre, 1919, En Lorraine. Petits croquis de l'arrière, Paris, Berger-Levrault.

\section{Sitographie}

http://www.vosges.fr/La-vie-en-Vosges/Tourisme-Vosges/Ligne-bleue.htm. Site consulté le 12 juin 2013.

http://www.deodatie.com/theme.html?id=126. Site consulté le 12 juin 2013. http://www.bravepatrie.fr/. Site consulté le 12 juin 2013.

\section{Références}

Amossy Ruth, 2000, L'argumentation dans le discours. Discours politique, littérature d'idées, fictions. Comment peut-on agir sur un public en orientant ses façons de voir, de penser?, Paris, Nathan.

BACOT Paul, RÉMI-GIRAUd Sylviane, 2002, «Présentation. Les métaphores spatiales en politique », Mots. Les Langages du politique, no 68.

BECKER Jean-Jacques, 2001, «1905: La menace de guerre est-elle à l'origine d'un renouveau nationaliste? ", Mil neuf cent. Revue d'histoire intellectuelle, $\mathrm{n}^{\circ} 19$.

Bon homme Marc, 2005, Pragmatique des figures du discours, Paris, Champion.

DuBoıs Danièle éd., 2009, Le sentir et le dire. Concepts et méthodes en psychologie et linguistique cognitives, Paris, L'Harmattan.

FEYEL Gilles, 2007, La presse en France des origines à 1944. Histoire politique et matérielle, Paris, Ellipses (Infocom).

Fumey Gilles, 2006, "Le Tour de France ou le vélo géographique », Annales de Géographie, nº650. 
Guilhaumou Jacques, 2006, Discours et événement. L'histoire langagière des concepts, Besançon, Presses universitaires de Franche-Comté.

JAmEt Denis, Jobert Manuel éd., 2010, Empreintes de l'euphémisme. Tours et détours, Paris, L'Harmattan.

KALIFA Dominique, 2001, La culture de masse en France, Paris, La Découverte (Repères).

KRIEg-Planque Alice, 2009, La notion de "formule» en analyse du discours. Cadre théorique et méthodologique, Besançon, Presses universitaires de Franche-Comté.

Maingueneau Dominique, 1998, Analyser les textes de communication, Paris, Dunod.

Molinier Christian, 2006, «Les termes de couleur en français. Essai de classification sémantico-syntaxique », Cahiers de grammaire, n³0, ERSS.

MORINIAUX Vincent, 2001, «Le mythe de la ligne bleue des Vosges et la vision nationaliste de la sapinière », Le Sapin, Enjeux anciens, enjeux actuels, Andrée Corvol éd., Paris, L'Harmattan.

Pastoureau Michel, 2002, Bleu, histoire d'une couleur, Paris, Le Seuil.

THIERRY Frédéric, " "La première veste de camouflage de guerre du monde” est inventée par Louis Guingot», Guerres mondiales et conflits contemporains, Paris, PUF.

STERNHELl Zeev, 1978, La droite révolutionnaire. Les origines françaises du fascisme, 1885-1914, Paris, Le Seuil (Points). 